\title{
Impact of differential surface anisotropy on Biopharmaceutical Performance of Celecoxib
}

\section{Poonam Singh Thakur $^{1}$, Sameer Ramanlal Modi ${ }^{1}$, Changquan Calvin Sun ${ }^{2}$, Arvind K Bansal ${ }^{1}$}

${ }^{1}$ Pharmaceutics, National Institute Of Pharmaceutical Education And Research, Sas, Mohali, India, ${ }^{2}$ Pharmaceutical Materials Science and Engineering Laboratory, Department of Pharmaceutics, College of Pharmacy, University of Minnesota, , United States, Minnesota, United States

E-mail: poonamsinghniper@gmail.com

Inconsistency in drug product quality poses significant challenges in formulation development of BCS class II drugs, since their performance is sensitive to material properties. Within this framework, vast knowledge base has been generated with respect to the impact of polymorphs / pseudopolymorphs / amorphous form on pharmaceutical performance of active pharmaceutical ingredients (APIs). However, the role of crystal habit has remained largely ignored. Anisotropic surface chemistry of different crystal facets in different crystal habits may also affect properties like kinetic solubility, dissolution behavior and in vivo performance. The present investigation deals with crystal habit modification of celecoxib (CLB) and its effect on biopharmaceutical performance. Two crystal habits namely Acicular shape (A-CLB) and Plate shape (P-CLB) of CLB were generated by recrystallization from toluene at $25^{\circ} \mathrm{C}$ and $60{ }^{\circ} \mathrm{C}$, respectively. P-CLB exhibited significantly faster dissolution kinetics and intrinsic dissolution rate in $\mathrm{pH} 12$ phosphate buffer compared to A-CLB. The oral bioavailability study showed significantly higher Cmax and shorter Tmax compared to A-CLB. The significant enhancement in biopharmaceutical performance of P-CLB was attributed to its more abundant hydrophilic surfaces than A-CLB. The surface anisotropy of both of the crystal habit was supported by wettability and surface free energy determination from contact angle measurements, surface chemistry determination by X-ray photoelectron spectroscopy (XPS), crystal structure modeling and crystal face indexation.

1. Modi, S. R.; Dantuluri, A. K. R.; Perumalla, S. R.; Sun, C. C.; Bansal, A. K. Cryst.

Growth Des. 2014, 14, 5283-5292.

2. Heng, J. Y. Y.; Williams, D. R. Langmuir 2006, 22, 6905-6909.

3. Sun, C.; Grant, D. J. W. J. Pharm. Sci. 2001, 90, 569-579.
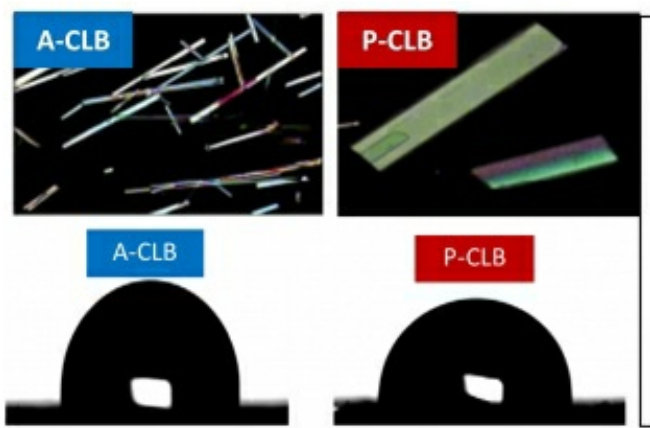

$95.28 \pm 2.23$

$86.88 \pm 2.67$

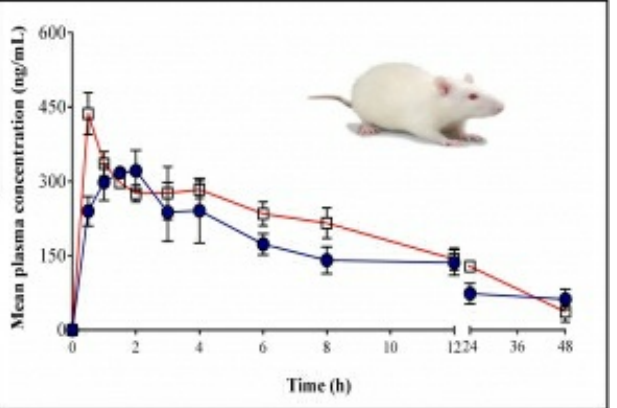

Keywords: Celecoxib, crystal habits, biopharmaceutical performance 